\title{
Infrared and tactile units in the sensory trigeminal system of python reticulatus
}

\section{G. J. MOLENAAR, J. L. F. P. FIZAAN-OOSTVEEN and J. M. VAN DER ZALM}

Institute of Veterinary Anatomy, and (J.v.d. Z.) Institute of Veterinary Pharmacology and Foxicology, University of Utrecht, 3572 SG Utrecht (The Netherlands)

(Accepted March 15th, 1979)

During the past decades the infrared sensitivity of certain snakes has received ample interest. Attention was paid to behavioral and ecological aspects, to the structure and function of the receptors and their trigeminal nerve branches as well as to responses at higher brain levels ${ }^{1,2,9}$. Previous neuroanatomical studies ${ }^{6,7,8}$ have shown that the sensory trigeminal nuclei of snakes with infrared sensitivity form two distinct systems. One of these systems closely resembles the sensory trigeminal system of other vertebrates and is referred to as the common sensory trigeminal system. Its nucleiform a longitudinal column of gray matter, which extends throughout the rhombencephalon and merges caudally with the dorsal horn of the cervical cord. The column can be subdivided into a number of nuclei and subnuclei, similar to those in mammals ${ }^{6}$. For the present study, the trigeminal principal sensory nucleus and the nucleus caudalis are the most relevant structures. The other system is situated laterally to the descending portion of the common sensory trigeminal system. It consists of descending primary sensory trigeminal fibres and a huge nucleus in which these fibres terminate. They are called the lateral descending trigeminal tract and nucleus (lttd and LTTD). The nucleus is macroscopically distinguishable by its protrusion from the lateral side of the medulla.

The presence of these two distinct sensory trigeminal systems had led us to assume that the general sensory modalities are relayed to the common sensory trigeminal nuclei, while radiant heat is conveyed to the lateral descending trigeminal nucleus. Comparative anatomical studies ${ }^{4}$, iontophoresis of cobalt chloride ${ }^{8}$ and electrophysiological techniques ${ }^{7,10}$ demonstrated the existence of a relation between the lateral descending nucleus and infrared sensitivity. In the present account we shall describe the responses upon infrared and tactile stimulation of a limited number of units in nuclei belonging to either of the two sensory trigeminal systems, in Python reticulatus.

The surgical procedure was carried out under fluothane anesthesia following premedication with metomidatum (Hypnodil, Janssen Pharmaceutica, Belgium, 8 $\mathrm{mg} / \mathrm{kg}$ i.p.). It involved reflection of the jaw muscles and the maxillary vein, removal of 
the outer table of the skull, the entire inner ear, the inner table of the skull and the dura mater. After this procedure the lateral side of the rhombencephalon was exposed from a level slightly rostral to the trigeminal nerve roots to the first cervical segment. Great care was taken to prevent damage to the rostral and caudal transverse blood sinuses which cover the brain areas over the nuclei principalis or caudalis, respectively. Damage to these vessels is fatal.

When the rhombencephalon was completely exposed, the fluothane anesthesia was stopped and local anesthesia by means of procaine was applied around the operation wound. The snake was immobilized by application of D-tubocurarine, 5 $\mathrm{mg} / \mathrm{kg}$ j.p. Every few hours, depending on the snake's reactions, this dose was repeated. A cannula was inserted into the glottis and artificial respiration was started, adjusted to the snake's natural respiratory volume. The body temperature was maintained at about $30^{\circ} \mathrm{C}$.

Activity was recorded from the nucleus of the lateral descending trigeminal tract as well as from the nucleus principalis and the nucleus caudalis of the common sensory trigeminal system. Recordings were made at various depths along electrode tracks which penetrated these nuclei from the lateral or dorsolateral side. The different sensory trigeminal nuclei could easily be recognized macroscopically due to their superficial position and protrusion from the lateral side of the brain. The medial extent of a nucleus at a certain level was determined stereo-taxically froi an atlas of the Python's brain 5 .

Glass microelectrodes, filled with $3 \mathrm{M} \mathrm{NaCl}$ and with a resistance of about $1 \mathrm{M} \Omega$, were used to make the recordings. The signal was amplified by an $\mathrm{AC}$ amplifier, displayed on an oscilloscope and recorded on magnetic tape simultaneously with the stimulus for further analysis.

Infrared stimulation was applied by means of a beam of parallel rays from an incandescent lightbulb $(6 \mathrm{~V}, 5 \mathrm{~A})$, that could be screened by means of a metal plate.

Stimulation of the general senses was accomplished by touching, pressing, scratching or pricking the various parts of the snake's head or body by means of cottonwool or a syringe needle.

Thirty-two single unit and 12 multiple unit recordings were made in 3 animals. Twenty units out of these were located in the lateral descending nucleus and responded to stimulation of the pit organs by infrared radiation. They responded neither to sounds nor to vibrations. Touching the face or the pits with cotton-wool or a needle sometimes evoked a response. However, it seems doubtful that also responses to mechanical stimuli are conveyed to the lateral nucleus. Usually a response was obtained before the skin was actually touched; in fact, the mere approach of the head appeared sufficient to evoke a response by changing the heat flux to the pits. This was most probably the real effective stimulus and not the touching of the skin.

Eight single units belonged to nuclei of the common sensory system, i.e. the nuclei principalis or caudalis. They responded to touching of the skin or teeth, but did not show any response to infrared stimulation; neither did 12 multiple units in these nuclei.

A crude somatotopic projection from the pit organs to the lateral descending 


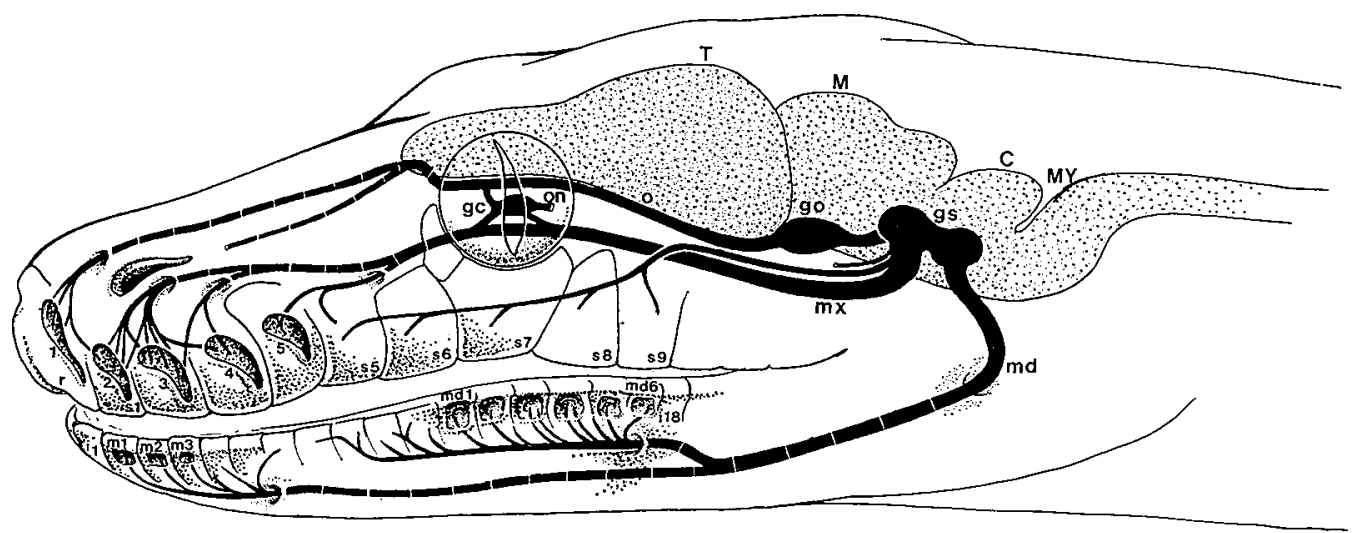

Fig. 1. Schematic drawing of the head of Python reticulatus, showing the distribution of the 3 trigeminal nerve branches to the various pit organs. The broken lines represent the passage of the nerves through bone. Note that distinct groups of pit organs are innervated by distinct nerve bundles. Maxillary pits are occasionally innervated by small fibre bundles which diverge from the bundies to neighbouring pits. $1-5$, maxillary pits; $I_{1}-I_{18}$, infralabial scales; $m_{1}-m_{3}$, mental pits; mdl-md6, mandibular pits; $S_{1}-S_{9}$, supralabial scales; $C$, cerebellum; gc, anastomosis between the maxillary and ophthalmic branches via the ciliary ganglion; go, ganglion in the ophthalmic branch; gs, semilunar ganglion; $M$, mesencephalon; $m$, mandibular branch of the trigeminal nerve; mx, maxillary branch of the trigeminal nerve; MY, myelencephalon; 0 , ophthlamic branch of the trigeminal nerve; on, oculomotor nerve; $r$, rostral scale; $\mathrm{T}$, telencephalon.

nucleus was found. It resembles the projection of the 3 peripheral trigeminal main branches into distinct territories of the lateral nucleus. Thus, the mandibular pits are represented in the dorsal part of the nucleus, the maxillary pits project intermediately and the rostral pits project to the ventral parts of the nucleus. However, a projection of a single pit to an individual unit or to a restricted area of the nucleus could not be demonstrated. Although a number of 14 units is not sufficient to draw reliable conclusions, it is remarkable that each of these units responded to stimulation of a certain number of pit organs. Dissection studies revealed that such a distinct group of pit organs is usually innervated by a distinct group of fibre bundles from the trigeminal nerve (Fig. 1).

The responses of units, connected with the mandibular pits, are represented in Fig. 2. The pits were stimulated by moving a $2 \mathrm{~mm}$ thick metal plate in a caudorostral

A

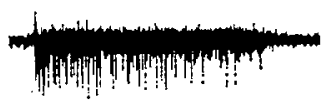

B

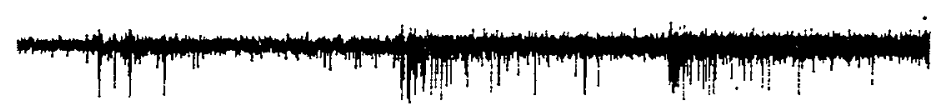

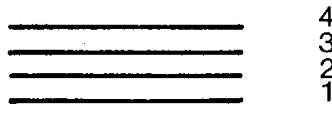

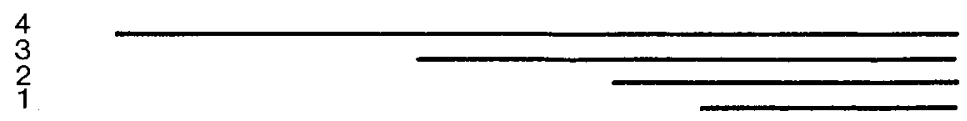

Fig. 2. Activity of multiple units in the lateral descending trigeminal nucleus connected with various mandibular pits. The presence of the stimulus is indicated by the lower trace. A: response to simultaneous stimulation of the mandibular pits. B: response to stimulation of individual pits. 
direction along the pits, while the pits 5 and 6 were permanently screened. A short response is visible after stimulation of pit 4 . When adaptation of pit 4 is accomplished, both pits 4 and 3 are exposed to stimulation, which results in another burst of spikes. The same phenomenon is seen when successively also pit 2 and pit I (so pits $4+3 \div 2$

1) are exposed. Similar responses were obtained from other units, which were connected to the pits 1 and 2 or to the pits $3,4,5$. One unit was driven by stimulation of both the supralabial scales L5, L6, L7 and the 3 or 4 infralabial scales immediately in front of the mandibular pits. Similar converging projections from neighbouring pits have been reported to exist for tectal units too. Their extent of convergence is proportional to the overlap of their receptive fields ${ }^{3}$.

The majority of the units in the LTTD showed a certain activity during the intervals between the applied stimuli. This activity was highly irregular and sometimes even disappeared for periods of several minutes to occasionally half an hour. During these silent periods, the units showed normal responses upon stimulation.

The responses of the units in the LTTD were greatly variable in duration and frequency. One of the most striking features was the shift from tonic to phasic responses upon repetitive stimulation at a constant intensity.

The units in the common sensory trigeminal nuclei showed similar irregularities in their responses and interval activity, but their fluctuations were less pronounced.

The present study demonstrates that in the sensory trigeminal system of Python there is a separation of the pathways for the perception of infrared and common trigeminal sensory modalities. This confirms our previous postulation that was based on comparative anatomical studies 4 . Thus, infrared data are relayed via the lateral descending trigeminal tract to the homonymous nucleus, while common trigeminal sensory modalities are relayed through the nuclei of the common sensory trigeminal system, i.e. the principal sensory and the caudal trigeminal nuclei. The number of units sampled in either of the two sensory trigeminal systems, however, is too small to rule out the possibility that the two systems mutually exchange information. Since a complete separation is present between both the primary and secondary projections $\mathrm{s}^{\mathrm{i}, 7}$ of the two systems and since not even the faintest sign of single or multiple unit activity was found in either of the two systems upon stimulation of the receptors of the other system, we consider this exchange as highly unlikely.

1 Barrett, R., The pit organs of snakes. In C. Gans and T. S. Parsons (Eds.), Biology of the Reptilia, Vol. 2, Academic Press, London, 1970, pp. 277-300.

2 Hartline, P. H., Thermoreception in snakes. In A. Fessard (Ed.), Handbook of Physiology, Vol. III/3, Electroreceptors and Other Specialised Receptors in Lower Vertebrates, Springer-Verlag, Berlin, New York, 1974, pp. 297-312.

3 Haseltine, E. C., Infrared and Visual Organization of the Tectum of Boid Snakes. Thesis, Indiana University, 1978.

4 Molenaar, G. J., An additional trigeminal system in certain snakes possessing infrared receptors, Brain Research, 78 (1974) 340-344.

5 Molenaar, G. J., The rhombencephalon of Python reticulatus, a snake possessing infrared receptors, Ned. J. Zool., 27 (1977) 133-180.

6 Molenaar, G. J., The sensory trigeminal system of a snake in the possession of infrared receptors. I. The sensory trigeminal nuclei, J. Comp. Neurol., 179 (1978) 123-136. 
7 Molenaar, G. J., The sensory trigeminal system of a snake in the possession of infrared receptors. II. The central projections of the trigeminal nerve, J. comp. Neurol., 179 (1978) 137-152.

8 Schroeder, D. M. and Loop, M. S., Trigeminal projections in snakes possessing infrared sensitivity, J. comp. Neurol., 169 (1976) 1-14.

9 Terashima, S. I. and Goris, R. C., Tectal organization of pit viper infrared reception, Brain Research, 83 (1975) 490-494.

10 Terashima, S. I. and Goris, R. C., Infrared bulbar units in Crotaline snakes, Proc. Jap. Acad., 53, Ser. B. (1977) 292-296. 